\title{
26 Research Square \\ SAPK2 contributes to rice yield by modulating nitrogen metabolic processes under drought stress
}

Dengji Lou

Yuxi Normal University

Xiaoyan Yang ( $\nabla$ yangxiaoyan9999@163.com )

The University of Manchester Faculty of Biology Medicine and Health https://orcid.org/0000-00020691-0406

Original article

Keywords: Rice, SAPK2, Grain yield, Grain size, NO3- influx, Drought stress, nutrient-deprived

Posted Date: March 28th, 2020

DOl: https://doi.org/10.21203/rs.3.rs-19566/v1

License: (1) This work is licensed under a Creative Commons Attribution 4.0 International License.

Read Full License

Version of Record: A version of this preprint was published at Rice on June 8th, 2020. See the published version at https://doi.org/10.1186/s12284-020-00395-3. 


\section{Abstract}

Background The sucrose non-fermenting 1-related kinases 2 (SnRK2s) play important roles in osmotic stress responses in A. thaliana and rice ( Oryza sativa L.). Osmotic stress/ABA-activated protein kinase 2 (SAPK2) is a member of SnRK2s subclass II in rice, but its function in rice yield under drought stress is unclear. Results Compared with wild-type (Oryza.Sativa L.spp.japonica, WT) plants, the sapk2 rice mutant lines were shorter and produced fewer grains per panicle, smaller grains and lower grain yield. Subsequent analysis suggested that SAPK2 considerably influences the nitrogen, phosphorus, and potassium contents of rice grains. The examination of rice seedling growth and development under nutrient-deprived conditions $(-\mathrm{N},-\mathrm{K}$, and $-\mathrm{P})$ proved that SAPK2 can significantly affect rice seedling growth and root development in hydroponic cultures lacking N and K. Moreover, the NO 3 - influx rate and nitrate concentration analysis indicated that SAPK2 promotes nitrate uptake and assimilation by regulating nitrate-related transporters. Conclusion These results suggest that SAPK2 could enhance grain production by regulating nitrogen utilization efficiency under drought stress. Our work provided insights to breeding drought tolerant rice with high nutrient uptake.

\section{Background}

The sustainability of crop production has been challenged by climate changes, an insufficient freshwater supply, and an increasing global population (Comas et al. 2013; Gray and Brady 2016). Additionally, drought stress, which is important abiotic factor limiting crop productivity worldwide, is responsible for extensive crop losses and will likely worsen in the near future. Thus, there is increasing international interest in developing drought-tolerant crops. In rice, plant architecture and grain yield are affected by environmental conditions and genetics. Enhancing the drought tolerance of rice is challenging because of the complexity of this trait and the incomplete characterization of the physiological and molecular mechanisms associated with drought responses. Accordingly, considerable work is required to address the adverse effects of drought stress and ensure the food security of future generations.

Drought stress can affect plants at all growth stages, and the extent of the changes to productivity depend on the plant species and its genotype, age, and size as well as the duration and severity of the stress (Gall et al. 2015). Plant growth is highly sensitive to water deficit, largely because of the resulting inhibition of cell elongation. Water-stressed plants are shorter than normal and have a smaller leaf area, which decreases the amount of photo synthetically active radiation absorbed by leaves, the photosynthetic rate, and ultimately yield. Water deficit induces stomata closure, which leads to limited $\mathrm{CO}_{2}$ uptake by leaves and decreased net photosynthesis (Yang et al. 2017). It also affects carbohydrates, the ATP content, respiration, and abscisic acid (ABA). Moreover, it leads to the excessive accumulation of reactive oxygen species, resulting in oxidative stress that seriously damages cellular functions (Deeba et al. 2012). In addition to its direct impact on plants, drought stress also has various indirect effects on crop growth and yield. Nutrients, especially macronutrients, are very important for plant growth and yield, and their uptake is restricted under drought stress conditions (Aroca 2012). The uptake of nitrogen (N) and potassium (K) reportedly decreases in cotton plants exposed to drought stress (McWilliams 2003). 
The concentration of growth-retarding substances usually increases under stress conditions to adjust plant water levels for various processes (Farooq et al. 2009).

Plant responses to drought stress include stress signal perception, signal transduction and amplification, and adaptations at the morphological, physiological, and molecular levels. Phytohormones are the key mediators of plant responses to drought stress (Sah et al. 2016). Water deficit also alters the endogenous synthesis of various phytohormones, including jasmonic acid (JA), ABA, salicylic acid (SA), ethylene (ET), auxin, gibberellins (GAs), and cytokinins (CKs). ABA is the major stress-responsive hormone produced after the drought signal is perceived by plants. Osmotic stress promotes ABA synthesis, which activates gene expression and adaptive physiological changes (Yamaguchi-Shinozaki and Shinozaki 2006). Additionally, ABA remains the best-studied hormone for plant stress responses, and the interaction between $A B A$ and other classical stress-related hormones enables plants to rapidly respond and properly adapt to drought stress (Brodribb and McAdam 2017). The ABA-dependent signaling pathways are critical for the expression of genes responsive to various stresses, especially osmotic stress. SnRK2 family members are protein kinases which promote ABA responses (Hrabak et al. 2003).

To date, 10 plant-specific SnRK2s have been identified in Arabidopsis thaliana (SnRK2.1-2.10) and rice (SAPK1-10) (Kobayashi et al., 2004). In A. thaliana, SnRK2.2, SnRK2.3, and SnRK2.6 are involved in ABA responses (Fujii and Zhu 2009). For example, upon hyperosmotic stress, ABA accumulates and binds to the PYR/PYL/RCAR-type ABA receptors, which subsequently inhibit PP2C activity, resulting in the release of SnRK2s from inhibition, and then the activated SnRK2s phosphorylate downstream effectors to mediate stress responses (Fujii et al. 2009; Ma et al. 2009; Park et al. 2009; Santiago et al. 2009; Umezawa et al. 2009). Additionally, in rice, members of SnRK2 subclass III (SAPK8-10), as well as SAPK2, help regulate ABA-dependent gene expression via the ABA signal transduction pathway mediated by OsPYL/RCARs and PP2Cs (Kim et al. 2012).

A recent study confirmed that the overexpression of SAPK9 may significantly enhance drought tolerance, while also increasing the grain yield under drought condition (Dey et al. 2016). In previous research, we found that the sapk2 mutants exhibited an ABA-insensitive phenotype during the germination and postgermination stages and sensitive phenotype to drought stress, with lower survival rates than the widetype plants. The mutants also exhibited greater water loss, lower proline and soluble sugar contents, higher proportions of fully open stomata, higher ROS levels, and lower antioxidant enzyme activities (Lou et al. 2017). These results indicate that SAPK2 may be useful for improving crop yields under drought conditions. However, the effect of SAPK2 on the productivity under drought stress remains unclear.

In this study, we examined knock-out mutant lines (sapk2; sapk2-1 and sapk2-7), which we previously developed with the CRISPR/Cas9 system, and SAPK2-overexpressing lines (OE; OES2-1 and OES2-2). We found that the sapk2 mutant showed lower grains yield and lower nitrogen, phosphorus, and potassium contents of rice grains than the WT under drought stress. Moreover, the sapk2 mutant exhibited weaker seedling growth and root development in hydroponic cultures lacking $\mathrm{N}$ and $\mathrm{K}$. The $\mathrm{NO}_{3}{ }^{-}$influx rate and nitrate concentration analysis indicated that SAPK2 promotes nitrate uptake and assimilation by 
regulating nitrate-related transporters. Our work provided insights to breeding drought tolerant rice with high nutrient uptake.

\section{Methods}

\section{Generation of transgenic rice lines}

We employed knock-out mutant lines (sapk2, sapk2-1 and sapk2-7) which we built previously by the CRISPR/Cas9 system and over-expression lines (OE, OES2-1 and OES2-2) (Lou et al. 2018). Concisely, the third coding exons of SAPK2 were selected for guide RNA design. Double-strand DNA generated by annealing the oligo pairs, and then was cloned into the PYLCRISPR/Cas9Pubi-H vector. For mutation detection, genomic DNA extracted from mutant seedlings (all plant) were used for PCR. In $T_{0}$ generation, we collected 20 hygromycin-resistant plants for each gene. Based on mutation detection results, we identified two independent homozygous mutant lines in the $\mathrm{T}_{1}$ generation, which we named sapk2-1, sapk2-7. To generate SAPK2 overexpression transgenic plants, the full-length cDNA of SAPK2 was cloned into the p1301 vector in the sense orientation behind the CaMV 35S promoter. Rice (Oryza sativa L. japonica.) was used for transformation. The primers used in this study were listed in the Additional file 2: Table S1.

\section{Plant cultivation and agronomic traits analysis}

For basic agronomic traits analysis, rice plants were grown in the paddy field from March to August at the rice experimental station of Xishuangbanna Tropical Botanical Garden. Ten plants at a spacing of 16.5 $\mathrm{cm} \times 26.5 \mathrm{~cm}$ were planted in a row and 5 rows of each line were planted. At reproductive stage, 20 plants of each line were randomly chosen to detect agronomic traits. The grain number per panicle was measured as the total number of grains per plant divided by the number of panicles per plant. The 1000grain weight was calculated as the weight of the total grains per plant and divided by the grain number, then converted to 1000-grain weight. Grain yield per plant was measured as the weight of total grains per plant.

For reproductive-stage drought stress, transplanted experiments were maintained as described by Sandhu et al. (2016). The drought stress was initiated at 32 days after transplanting. After the inception of the stress, the soil water potential was measured using tensiometers (30 cm depth). The plots in the reproductive stage drought stress treatments were rewatered when the soil water potential dropped to -50 to $-70 \mathrm{kPa}$ (tensiometer). The decline in water table depth was measured on a daily basis with a meter scale inserted into a 1.1-m polyvinyl chloride pipe in the experimental fields at regular intervals in all RS treatments. The pipes were placed at 1.0-m depth with $10 \mathrm{~cm}$ of pipe remaining above the soil surface. The plots were rewatered when water table level reached $100 \mathrm{~cm}$ below the soil surface and most lines were wilted and exhibited severe leaf drying.

To analyze SAPK2 function in seedling growth and development under different nutrient-deprived conditions, seedlings at 7 DAG were cultured in basic nutrient solution $(\mathrm{pH}=5.8)$ for a week. Then 
seedlings at 14 DAG were transferred to nutrient-deprived solutions. Each nutrient solution was renewed every three days. Daytime conditions in the greenhouse were $32^{\circ} \mathrm{C}$, with light from a sodium lamp (400 W) for $14 \mathrm{~h}$; night-time conditions were $25^{\circ} \mathrm{C}$, and dark for $10 \mathrm{~h}$. At $34 \mathrm{DAG}$, root length, shoot length, root number and dry weight of each lines were measured.

\section{Measurement of nitrate, phosphorus, potassium and total nitrogen content and nitrate influx}

For free $\mathrm{NO}_{3}{ }^{-}$analysis, $0.1 \mathrm{~g}$ plant materials from different genotypes were homogenized by grinding in cold extraction buffer [50 mM Tris- $\mathrm{HCl}(\mathrm{pH} 7.0), 10 \mathrm{mM}$ imidazole, and $0.5 \%$ (w/v) b-mercaptoethanol]; the homogenates were then centrifuged at $12,000 \mathrm{~g}$ for $20 \mathrm{~min}$ at $4{ }^{\circ} \mathrm{C}$ and the supernatant was collected. Free $\mathrm{NO}_{3}{ }^{-}$in the supernatant was determined by using the Griess method (Walther et al. 1999), the absorbance at $540 \mathrm{~nm}$ was determined and $\mathrm{NO}_{3}$ contents were calculated from the standard curve of $\mathrm{KNO}_{3}$.

$\mathrm{NO}_{3}{ }^{-}$influx was calculated as the difference in $\mathrm{NO}_{3}{ }^{-}$content between the plants under control conditions and drought stress conditions in an hour.

For total nitrogen, phosphorus and potassium content analysis, 50 seeds from different genotypes were homogenized by grinding in Liquid nitrogen. Then $0.1 \mathrm{~g}$ seed materials were measured using Agilent 7700e ICP-MS.

At least three independent biological experiments were conducted. One representative result was displayed here.

\section{RNA extraction and qRT-PCR analysis}

To detect the transcript level of SAPK2 under different nutrient-deprived conditions, 14 DAG seedlings were transferred to different nutrient-deprived solutions for 24 hours. To detect the transcript level of target genes under drought stress, seedlings were transferred into half-strength liquid medium supplemented with $25 \%$ PEG6000 (m/v) for 24 hours. All samples were collected at the right time.

For the qRT-PCR analysis, we used the same method as described (Jiang et al. 2016). Total RNA was isolated from whole seedlings using the TriZol reagent (Invitrogen). The cDNAs were obtained by using Superscript II in accordance with manufacturer's instructions (Invitrogen). The qRT-PCR analysis was performed using SYBR Premix Ex Taq kit (Takara).

At least three independent biological experiments were conducted (three independent samples were conducted for each experiment and three technological replications in every independent experiment). One representative result was displayed here. Gene-specific primers used in qRT-PCR analysis were listed in Additional file 1: Table S1.

\section{Statistical analysis}


The experiments were arranged in a completely randomized design with at least three replicates for each treatment. Excel 2010 was used for making charts. Two-tailed Student's $t$ tests were performed using the SPSS 10 software (IBM, Inc.). "* and **” indicate significance at $P<0.05$ and $P<0.01$, respectively. The data represent mean \pm standard error $(\mathrm{SE})$ of three independent experiments.

\section{Results}

\section{Mutations to SAPK2 decrease plant height and grain yield}

Under drought conditions, the two sapk2 lines were shorter than the WT control plants (Fig. 1a-c). To further investigate the SAPK2 roles influencing rice yield, we analyzed OE lines (OES2-1 and OES2-2) and sapk2 knock-out mutant lines (sapk2-1 and sapk2-7). An analysis of plants exposed to reproductive stage drought stress (RS) indicated that the two sapk2 lines had substantially more tillers than the WT plants (Fig. 1a, d), whereas there were no significant differences in the two OE lines (Fig. 1b-d). However, although they had more tillers, the sapk2 mutant plants produced fewer grains per plant than WT under drought condition (Fig. 1f). A subsequent investigation of the regulatory effect of SAPK2 on the number of effective tillers revealed that the sapk2 mutant lines had considerably fewer effective tillers than WT, but there were no significant differences in the $O E$ lines (Fig. 1e). This result further confirmed that the sapk2 mutant produces fewer grains than WT under drought condition.

The number of grains per panicle is one of the three key factors determining rice grain yield (Xing and Zhang 2010). Thus, we investigated the SAPK2 roles related to panicle and grain development by analyzing the number of grains per panicle, the seed setting rate, the grain length and width, the 1,000grain weight and the grain yield per plant in response to RS. Under RS, the panicles and grains of the sapk2 mutant lines were smaller than those of WT (Fig. 2a). Additionally, the grain number per panicle of the sapk 2 mutant lines was $75 \%$ and $60.8 \%$ of that of WT and $O E$ lines (Fig. $2 \mathrm{~b}$ ). The setting rate of $O E$ lines did not differ from that of WT, whereas the setting rate of the sapk2 mutant lines decreased significantly (76.9\% of that of WT) (Fig. 2c). Compared with WT, the grains of $O E$ lines were significantly longer, whereas there was no significant difference in the grain length of the sapk2 mutant lines (Fig. 2d). In contrast, the grains of the sapk2 mutant lines were significantly thinner than WT, whereas the grain width of $O E$ lines was not significantly different (Fig. 2e). Moreover, the 1,000-grain weight and grain yield per plant of the sapk2 mutant lines were much lower than WT and OE plants (Fig. 2f, g). These results implied that SAPK2 influences panicle and grain sizes in rice.

Overall, our observations indicated that knocking out of SAPK2 significantly decreases rice plant height and grain yield per plant under drought condition. Additionally, overexpressing SAPK2 does not appear to enhance rice plant growth or grain production.

Mutations to SAPK2 decrease nitrate, phosphorus, and potassium contents in rice grains under reproductive-stage drought 
Umezawa et al. (2004) reported that the expression of SnRK2.8, which is a homolog of rice SAPK2, is down-regulated by potassium deprivation. This down-regulation is associated with a substantial decrease in the growth of $A$. thaliana under nutrient-deprived conditions. As mentioned earlier, SAPK2 influences rice panicle and grain sizes. To clarify the mechanisms by which SAPK2 influences panicle and grain sizes in rice, we measured total nitrogen, nitrate, phosphorus, and potassium contents of seeds. Under RS conditions, the seeds of the sapk2 mutant lines had lower total nitrogen, nitrate, phosphorus, and potassium content than WT, with the biggest difference observed for the total nitrogen (Fig. 3a-C, SFig 1). However, the total nitrogen, nitrate, phosphorus, and potassium content were relatively consistent between the $O E$ and WT plants (Fig. 3a-c).

Next, we investigated the SAPK2 expression profiles under control conditions (i.e., sufficient nutrients) and nutrient-deficient conditions [i.e., lacking $\mathrm{K}(-\mathrm{K}), \mathrm{N}(-\mathrm{N})$, and $\mathrm{P}(-\mathrm{P})$ ]. The qRT-PCR analyses revealed that the SAPK2 transcript levels in the roots decreased in the absence of N, $P$, and K (Fig. $3 d-f$ ).

These findings confirmed that in rice, the seed nitrate, phosphorus, and potassium contents are largely affected by SAPK2. Therefore, we hypothesized that SAPK2 influences panicle and grain sizes by modulating metabolic processes of $\mathrm{N}, \mathrm{P}$, and $\mathrm{K}$.

\section{SAPK2 affects seedling growth and root development in response to $\mathrm{N}$ and $\mathrm{K}$ deprivation}

To further validate our hypothesis, we investigated the effects of knocking out and overexpressing SAPK2 on rice seedling growth and development in hydroponic cultures under different nutrient-deprived $(-\mathrm{K},-\mathrm{N}$, and $-\mathrm{P}$ ) conditions.

The sapk2 mutant seedlings under N-deprived conditions produced weaker culms than WT, whereas the $O E$ lines were phenotypically similar to WT (Fig. 4a, f; In Fig. 4f, the $O E$ phenotype is not presented). A previous study proved that the root morphology influences plant interactions with soil nitrates, making it important for $\mathrm{N}$ absorption (Hachiya and Sakakibara 2017). Accordingly, we examined the root development of the SAPK2 transgenic lines. In the sapk2 mutant lines, root growth was inhibited, resulting in roots than WT seedlings (Fig. 4b, f). In contrast, the root phenotypes of the $O E$ and WT plants were similar (Fig. 4b, f; In Fig. 4f, OE phenotype is not presented). The root and shoot dry weights of the sapk2 mutant lines were significantly lower than WT, but there were no significant differences in the $O E$ lines (Fig. 4c, d). Similarly, compared with WT, the sapk2 mutant lines had fewer roots, whereas there were no significant differences in the $O E$ lines (Fig. 4e).

The effects of the K-deprived conditions were similar to those of the N-deprived conditions. For example, the sapk2 mutant seedlings produced weaker culms and had lower root and shoot dry weights than WT (SFig. 2a-f). In contrast, the exposure to P-deprived conditions did not result in any significant differences in the seedling growth and root development of the WT, OE and sapk2 seedlings (SFig. 3a-f).

These findings suggested that SAPK2 can significantly influence rice seedling growth and root development in hydroponic cultures under $\mathrm{N}$ - and K-deprived conditions. 


\section{SAPK2 influences the $\mathrm{NO}_{3}{ }^{-}$influx rate and nitrate concentration under drought stress condition}

To explore the potential mechanism underlying the effects of SAPK2 on rice seedling growth and root development under $\mathrm{N}$-deprived condition, we investigated the $\mathrm{NO}_{3}{ }^{-}$influx rate and nitrate concentration of the WT, $O E$, and sapk2 plants under control and drought conditions. Under control conditions, there were no significant differences among the WT, $O E$, and sapk2 plants (Fig. $5 \mathrm{a}, \mathrm{c}$ ). However, in response to drought stress, the $\mathrm{NO}_{3}{ }^{-}$influx rate and nitrate concentration significantly decreased in the WT, $O E$, and sapk2 plants (Fig. 5a-d). Additionally, the rate of $\mathrm{NO}_{3}{ }^{-}$influx into the roots was lower for the sapk2 mutant lines than for WT (Fig. 5b), suggesting that knocking out of SAPK2 weakens the nitrate uptake by the roots. Regarding the sapk2 mutant lines, we also detected a lower rate of $\mathrm{NO}_{3}{ }^{-}$influx into the leaf sheath and leaf blade, implying that SAPK2 promotes the translocation of $\mathrm{NO}_{3}{ }^{-}$from the roots to the leaf sheath (Fig. 5b). Moreover, the root, leaf sheath, and leaf blade nitrate concentrations were consistent with the $\mathrm{NO}_{3}{ }^{-}$influx rates in the different lines (Fig. $5 \mathrm{~d}$ ). These results demonstrated that SAPK2 enhances nitrate influx and increases the nitrate concentration by promoting the translocation of nitrate from the roots to the leaf sheath.

Nitrogen use efficiency is an important trait for the development of sustainable agricultural production (Xu et al. 2012). Plants have diverse transporters facilitating $\mathrm{N}$ uptake and internal distribution (Rentsch et al. 2007). In higher plants, members of the NPF family (previously called the PTR/NRT1 family) can take up and translocate nitrate or small peptides. Of the rice NPF family members, only a few have been studied. For example, OSNPF7.2, which encodes a positive regulator of nitrate influx and concentration, helps control the allocation of nitrate between the roots and shoots (Wang et al. 2018). In rice, the peptide transporter OsNPF7.3 (OsPTR6) mediates the transport of organic $\mathrm{N}$ from the leaves to the grains and increases the grain yield (Fang et al., 2017). Additionally, OsNPF6.5 (OsNRT1.1B) is predominantly expressed in the root hairs, epidermis, and stellar cells adjacent to the xylem in roots. The osnrt1.1b mutant is reportedly defective in both nitrate uptake and root-to-shoot nitrate transport, suggesting that OsNRT1.1B is involved in nitrate uptake and transport (Hu et al., 2015). Down-regulating OsNRT2.3a expression impairs the loading of nitrate into the xylem and inhibits plant growth under low-nitrate conditions, implying OsNRT2.3a contributes to the long-distance transport of nitrate from the roots to the shoots (Tang et al., 2012). The silencing of OsNPF2.4 diminishes the low-affinity nitrate acquisition by roots, disrupts the K-coupled root-to-shoot nitrate transport, and inhibits the redistribution of nitrate from old leaves to N-starved roots or young leaves (Xia et al., 2015).

To further validate our hypothesis that SAPK2 influences panicle and grain sizes by modulating $\mathrm{N}$ metabolic processes, we determined the expression levels of genes crucial for the absorption, transport, and assimilation of nitrate among the WT, $O E$, and sapk2 plants cultured under control and drought conditions. The OsNPF7.2, OsNPF7.3, OsNPF5.6, OsNPF2.2, OsNRT2.3a, and OsNPF2.4 expression levels were significantly lower in the sapk2 mutant lines than in WT under drought condition (Fig. 6a-f). However, the opposite expression patterns were detected for the OE lines (Fig. 6a-f). These results implied that SAPK2 promotes nitrate uptake and assimilation by regulating nitrate-related transporters. 


\section{Discussion}

\section{Mutations to SAPK2 decrease grain yield}

Drought is one of the most important environmental stresses affecting the productivity of most field crops (Gray and Brady 2016). Elucidating the complex mechanisms underlying the drought resistance of crops will accelerate the development of new varieties with enhanced drought resistance. Improving the grain yield is the primary aim for most rice breeders. The rice yield potential is determined by the biomass and harvest index.

To identify additional rice grain yield-related genes affected by drought stress, we functionally characterized SAPK2 by examining sapk2 mutant lines exposed to drought stress. Under RS condition, the sapk2 mutant plants produced fewer effective tillers and grains per plant than the WT and $O E$ plants. Rice grain yield is determined by the following three traits: number of panicles, number of grains per panicle, and grain weight (Xing and Zhang 2010). Consequently, we were interested in whether SAPK2 can be used to improve rice yields under RS conditions. We also investigated the SAPK2 roles associated with panicle and grain development in response to RS conditions. Specifically, the panicle size, number of grains per panicle, grain size, setting rate, 1,000-grain weight and grain yield per plant were significantly lower for the sapk2 mutant lines than WT (Fig. 2a-g). These results indicated that SAPK2 increases rice yield under RS condition by influencing panicle and grain size.

\section{SAPK2 affects seedling growth and root development in response to $\mathrm{N}$ and $\mathrm{K}$ deprivation}

A previous study revealed that SRK2C/SnRK2.8 may mediate the phosphorylation of enzymes involved in metabolic processes (Umezawa et al., 2004). To investigate whether the decrease in the grain yield of the sapk2 mutant lines under RS conditions is related to nutrient metabolism, we measured the seed total nitrogen, nitrate, phosphorus, and potassium content. The data indicated the sapk2 mutant seeds had lower total nitrogen, nitrate, phosphorus, and potassium content than that in WT and OE lines seeds, especially for total nitrogen content (Fig. 3a-c and SFig. 1).

The roots, which are responsible for the uptake of water and nutrients from the soil, are vital for plant growth, development, and fitness. Additionally, ABA controls several key steps associated with lateral root initiation as well as meristem activation and elongation (De Smet et al. 2003; Ding and De Smet 2013). Root development is directly affected by environmental factors. Furthermore, the plant root system architecture is plastic and dynamic; enabling plants to respond to environmental changes, which then promotes root growth and development to avoid water deficit stress in the early stages of drought stress. Nitrogen availability is a major determinant of plant growth and crop productivity (Hachiya and Sakakibara 2017; Li et al. 2017). Plants use inorganic forms in natural soils, including nitrates, nitrites, and ammonium, and nitrate is the major form of $\mathrm{N}$ in most aerated soils. Of these available $\mathrm{N}$ sources, researchers have mainly focused on nitrate and ammonium because these are often present in natural and cropland soils at much higher levels than the other $\mathrm{N}$ sources (Miller and Cramer, 2004). 
To further validate the relationship between SAPK2 and N metabolism, we investigated the effects of knocking out and overexpressing SAPK2 on rice seedling growth and development in hydroponic cultures under $\mathrm{N}$-deprived conditions. The sapk2 mutant seedlings under $\mathrm{N}$-deprived conditions had weaker culms than WT, with inhibited root growth, significantly lower root and shoot dry weights, and fewer roots (Fig. 4a-f). However, we did not find significant difference between WT and OE plants (Fig. 4a-e).

Earlier investigations confirmed the importance of $\mathrm{K}$ for enzyme activities and ionic homeostasis in plants (Shabala and Pottosin 2014; Ahmad and Maathuis 2014). Additionally, we previously determined that SAPK2 regulates the expression of genes related to $\mathrm{Na}^{+}$and $\mathrm{K}^{+}$homeostasis, including OsSOS1, OsNHX1, OsHKT1;1, and OsHKT1;5 (lou et al. 2018). In this study, the sapk2 mutant seedlings deprived of $\mathrm{K}$ produced weaker culms and had lower root and shoot dry weights than WT and OE plants (SFig. 2).

Furthermore, we also investigated SAPK2 effects on rice seedling growth and development in hydroponic cultures under P-deprived conditions and found there was no significant difference between sapk2 mutant, WT and OE plants (SFig. 3).

Therefore, above results presented here confirm that SAPK2 influences panicle and grain sizes via its effect on $\mathrm{N}$ and $\mathrm{P}$ metabolic processes and is positive regulator in response to $\mathrm{N}$ and $\mathrm{K}$ deprived conditions.

However, the SAPK2 expression was down-regulated in response to $\mathrm{N}, \mathrm{P}$, and $\mathrm{K}$ deprivation in the roots (Fig. $3 d-f$ ). We think the reason for this result is related to how SAPK2 works. SAPK2 is a member of SnRK2 subclass II in rice. In the absence of ABA or osmotic stress, SAPK2is inhibited by ABA receptors OsPYL/RCAR5 and induces complex formation with PP2C30 through dephosphorylating. Under ABA or osmotic stress, ABA accumulates and binds to its receptors OsPYL/RCAR5, subsequently inhibit PP2C30 activity, resulting in the release of SAPK2 from inhibition. Activated SAPK2 phosphorylate downstream effectors to mediate stress responses (Kim et al. 2012). Current models suggest that ABA, which is induced by drought stress, inhibits the activity of OsPP2C49 to release the kinase activity of SAPK2 for further activation of OsbZIP23 through phosphorylation. OsbZIP23 could directly bind to the promoters of OsPP2C49 and positively regulates the expression of OsPP2C49, which in turn negatively regulates the ABA signaling (Zong et al. 2016). This regulatory mechanism of SAPK2 mainly occurs at the protein level, but does not change much at SAPK2 transcription level. However, there may be a negative regulatory mechanism at SAPK2 transcription level after this response, resulting in a decrease in SAPK2 transcription level.

\section{SAPK2 influences the $\mathrm{NO}_{3}{ }^{-}$influx rate and nitrate concentration}

Nitrogen uptake, assimilation, and recycling in plant roots reportedly determine plant development and productivity (Yamaya and Kusano, 2014). Plant growth under natural conditions is often limited by $\mathrm{N}$ availability. Therefore, plants have developed transport and signaling mechanisms for their specific $\mathrm{N}$ sources (Kiba and Krapp, 2016). To further validate the relationship between SAPK2 and nitrogen absorption and utilization by investigating the effects of silencing and overexpressing SAPK2 on rice. 
Under drought stress conditions, in the sapk2 mutant lines, we detected a lower rate of $\mathrm{NO}_{3}{ }^{-}$influx into roots and also into the leaf sheath and leaf blade (Fig. 5b). Besides, the detected nitrate concentration was consistent with the rate of $\mathrm{NO}_{3}{ }^{-}$influx in different lines (Fig. $5 \mathrm{~d}$ ). These results demonstrated that SAPK2 enhanced nitrate influx and concentration by promoting nitrate uptake by roots and translocation of nitrate from roots to leaf sheath.

Although there are more than 80 NRT1/PTR, 4 NRT2, and 2 NAR2 members in rice, only a few NRT1/PTR family members have been characterized (Araki and Hasegawa, 2006; Cai et al. 2008; Feng et al. 2011). Additionally, only a few nitrate transporters (OsNPF7.2, OsNPF7.3, OsNPF6.5, OsNPF2.2, OsNRT2.3a, and OsNPF2.4) have been characterized in rice (Wang et al. 2018; Fang et al. 2017; Hu et al. 2015; Li et al. 2015; Tang et al. 2012; Xia et al. 2015).

In this study, we analyzed the expression of several nitrate transporter genes (OsNPF7.2, OsNPF7.3, OsNPF5.6, OsNPF2.2, OsNRT2.3a, and OsNPF2.4). Under drought conditions, the expression levels of these six genes were significantly lower in the sapk2 mutant lines than in the WT plants in response to drought stress (Fig. 6a-f). Furthermore, OsNPF6.5 regulates the number of rice tillers and promotes grain production (Hu et al. 2015). The overexpression of OsNPF7.2 and OsNPF7.3 increases the production of tillers, panicles per plant, and filled grains per panicle, while also increasing the grain $\mathrm{N}$ content (Wang et al., 2018; Fang et al., 2017). The osnpf2.2 (OsPTR2) mutants are defective in long-distance nitrate transport, with repressed nitrate unloading from the xylem, resulting in plant growth retardation and abnormal grain filling (Li et al., 2015). Our study revealed that the sapk2 mutant lines produced significantly fewer tillers than the WT plants (Fig. 1d). Moreover, the sapk2 mutant seeds had a lower total nitrogen content than WT seeds under RS condition (Fig. 3a).

On the basis of our results, we conclude that under drought stress, SAPK2 promotes nitrate uptake and assimilation and influences the number of tillers and the number of grains per panicle by regulating nitrate-related transporters.

\section{Conclusions}

In this study, we examined knock-out mutant lines and SAPK2-overexpressing lines. We found that the sapk2 mutant showed lower grains yield and lower nitrogen, phosphorus, and potassium contents of rice grains than the WT under drought stress. Moreover, the sapk2 mutant exhibited weaker seedling growth and root development in hydroponic cultures lacking $\mathrm{N}$ and $\mathrm{K}$. The $\mathrm{NO}_{3}{ }^{-}$influx rate and nitrate concentration analysis indicated that SAPK2 promotes nitrate uptake and assimilation by regulating nitrate-related transporters. These results suggest that SAPK2 could enhance grain production by regulating nitrogen utilization efficiency under drought stress. Our work provided insights to breeding drought tolerant rice with high nutrient uptake.

\section{Abbreviations}


SAPK2: osmotic stress/ABA-activated protein kinase 2; S2-1:sapk2-7; S2-7:sapk2-7; OE: Overexpression; WT: Wild type; ROS: reactive oxygen species; JA: jasmonic acid; ABA: abscisic acid; SA: salicylic acid; ET: ethylene; Gas: gibberellins; CKs: cytokinins; RS: reproductive-stage drought; qRT-PCR: Quantitative Reverse Transcription Polymerase Chain Reaction; DAG: Days after germination

\section{Declarations}

\section{Availability of data and materials}

All data generated or analyzed during this study are included in this published article and its additional files.

\section{Acknowledgements}

Not applicable.

\section{Funding}

This research was supported by National Natural Science Foundation of China (31960371); National Natural Science Foundation of China (21562029).

\section{Authors' contributions}

D. J. L., X. Y. Y. planned and designed the research; D. J. L., and Z. C. performed the experiments; D. J. L. wrote the manuscript; X. Y. Y. analyzed the data and edited the article. All authors read and approved the final article.

\section{Ethics approval and consent to participate}

Not applicable.

\section{Consent for publication}

Not applicable.

\section{Competing interests}

The authors declare that they have no competing interests.

\section{References}

Ahmad I, Maathuis F (2014) Cellular and tissue distribution of potassium: physiological relevance, mechanisms and regulation. Plant Physiol. 171: 708-714 
Araki R, Hasegawa H (2006) Expression of rice (Oryza sativa L.) genes involved in high-affinity nitrate transport during the period of nitrate induction. Breeding Science 56: 295-302

Aroca R (2012) Plant responses to drought stress. In: From Morphological to Molecular Features. Springer-Verlag $\mathrm{GmbH}$, Berlin Heidelberg

Brodribb TJ, McAdam SA (2017) Evolution of the stomatal regulation of plant water content. Plant Physiol. 174:639-649

Cai C, Wang JY, Zhu YG, Shen QR, Li B, Tong YP, Li ZS (2008) Gene structure and expression of the highaffinity nitrate transport system in rice roots. Journal of Integrative Plant Biology 50: 443-451

Comas LH, Becker SR, Cruz VMV, Byrne PF, Dierig DA (2013) Root traits contributing to plant productivity under drought. Frontiers in Plant Science 4: 442.doi:10.3389/fpls.2013.00442

Deeba F, Pandey AK, Ranjan S, Mishra A, Singh R, Sharma YK, Shirke PA, Pandey V (2012) Physiological and proteomic responses of cotton (Gossypium herbaceum L.) to drought stress. Plant Physiol Biochem. 53: 6-18

De Smet I, Signora L, Beeckman T, Inzé D, Foyer C H, Zhang H (2003) An abscisic acidsensitive checkpoint in lateral root development of Arabidopsis. Plant J 33: 543-555

Ding Z, De Smet I (2013) Localised ABA signalling mediates root growth plasticity. Trends Plant Sci. 18: 533-535

Fang Z, Bai G, Huang W, Wang Z, Wang X, Zhang M (2017) The rice peptide transporter OsNPF7.3 is induced by organic nitrogen, and contributes to nitrogen allocation and grain Yield. Frontiers in Plant Science 8. doi:10.3389/fpls.2017.01338

Farooq M, Basra SMA, Wahid A, Ahmad N, Saleem BA (2009) Improving the drought tolerance in rice (Oryza sativa L.) by exogenous application of salicylic acid. Journal of Agronomy and Crop Science, 195(4):237-246

Feng H, Yan M, Fan X, Li B, Shen Q, Miller AJ, Xu G (2011) Spatial expression and regulation of rice highaffinity nitrate transporters by nitrogen and carbon status. Journal of Experimental Botany 62: 23192332

Fujii H, Chinnusamy V, Rodrigues A, Rubio S, Antoni R, Park S Y, Zhu JK (2009) In vitro reconstitution of an abscisic acid signaling pathway. Nature 462: 660-664

Fujii H, Zhu JK (2009) Arabidopsis mutant deficient in 3 abscisic acid activated protein kinases reveals critical roles in growth, reproduction and stress. Proceedings of the National Academy of Sciences 106(20):8380-8385 
Gall HL, Philippe F, Domon JM, Gillet F, Pelloux J, Rayon C (2015) Cell wall metabolism in response to abiotic stress. Plants 4(1): 112-166

Gray SB, Brady SM (2016) Plant developmental responses to climate change. Developmental Biology 419(1): 64-77

Hachiya T, Sakakibara H (2016) Interactions between nitrate and ammonium in their uptake, allocation, assimilation, and signaling in plants. Journal of Experimental Botany 68(10): 2501-2512

Hu B, Wang W, Ou S, Tang J, Li H, Che R, Chu C (2015) Variation in NRT1.1B contributes to nitrate-use divergence between rice subspecies. Nature Genetics 47(7): 834-838

Jiang YJ, Qiu YP, Hu YR, Yu DQ (2016) Heterologous expression of AtWRKY57 confers drought tolerance in Oryza sativa. Frontiers in Plant Science 7:145. doi: 10.3389/fpls.2016.00145

Kiba T, Krapp A (2016) Plant nitrogen acquisition under low availability: regulation of uptake and root architecture. Plant \& Cell Physiology 57, 707-714

Kim H, Hwang H, Hong JW, Lee YN, Ahn IP, Yoon IS, Kim BG (2012) A rice orthologue of the ABA receptor, OsPYL/RCAR5, is a positive regulator of the ABA signal transduction pathway in seed germination and early seedling growth. Journal of Experimental Botany 63(2): 1013-1024

Kobayashi Y, Yamamoto S, Minami H, Kagaya Y, Hattori T (2004) Differential activation of the rice sucrose nonfermenting1-related protein kinase2 family by hyperosmotic stress and abscisic acid. Plant Cell 16: 1163-1177

Li H, Hu B, Chu C (2017) Nitrogen use efficiency in crops: lessons from Arabidopsis and rice. Journal of Experimental Botany 68:2477-2488

Li Y, Ouyang J, Wang YY, Hu R, Xia K, Duan J, Wang Y, Tsay YF, Zhang M (2015) Disruption of the rice nitrate transporter OsNPF2.2 hinders root-to-shoot nitrate transport and vascular development. Scientific Reports 5(1): 9635. doi:10.1038/srep09635

Lou DJ, Wang HP, Liang G, Yu DQ (2017) OsSAPK2 confers abscisic acid sensitivity and tolerance to drought stress in rice. Frontiers in Plant Science 8: 993. doi:10.3389/fpls.2017.00993

Lou DJ, Wang HP, Yu DQ (2018) The sucrose non-fermenting-1-related protein kinases SAPK1 and SAPK2 function collaboratively as positive regulators of salt stress tolerance in rice. BMC Plant Biology 18(1): 203.doi:10.1186/s12870-018-1408-0

Ma Y, Szostkiewicz I, Korte A, Moes D, Yang Y, Christmann A, Grill E (2009) Regulators of PP2C phosphatase activity function as abscisic acid sensors. Science 324: 1064-1068 
McWilliams D (2003) Drought strategies for cotton, cooperative extension service circular 582. College of Agriculture and Home Economics, New Mexico State University, USA

Miller AJ, Cramer MD (2004) Root nitrogen acquisition and assimilation. Plant and Soil 274: 1-36

Park S, Fung P, Nishimura N, Jensen DR, Fujii H, Zhao Y, Cutler SR (2009) Abscisic acid inhibits type 2C protein phosphatases via the PYR/PYL family of START proteins. Science 324: 1068-1071

Rentsch D, Schmidt S, Tegeder M (2007) Transporters for uptake and allocation of organic nitrogen compounds in plants. FEBS Lett. 581: 2281-2289

Sah SK, Reddy KR, Li JX (2016) Abscisic acid and abiotic stress tolerance in crop plants. Frontiers in Plant Science 7: 571. doi: 10.3389/fpls.2016.00571

Sandhu N, Raman KA, Torres RO, Audebert A, Dardou A, Kumar A, Henry A (2016) Rice root architectural plasticity traits and genetic regions for adaptation to variable cultivation and stress conditions. Plant Physiology 171: 2562-2576

Santiago J, Dupeux F, Round A, Antoni R, Park SY, Jamin M, Marquez JA (2009) The abscisic acid receptor PYR1 in complex with abscisic acid. Nature 462: 665-668

Shabala S, Pottosin I (2014) Regulation of potassium transportin plants under hostile conditions: implications for abiotic and biotic stress tolerance. Physiologia Plantarum 151(3): 257-279

Tang Z, Fan X, Li Q, Feng H, Miller AJ, Shen Q, Xu G (2012) Knockdown of a rice stelar nitrate transporter alters long-distance translocation but not root influx. Plant Physiology 160: 2052-2063

Umezawa T, Yoshida R, Maruyama K, Yamaguchi-Shinozaki K, Shinozaki K (2004) SRK2C, a SNF1 related protein kinase 2 , improves drought tolerance by controlling stress-responsive gene expression in Arabidopsis thaliana. Proceedings of the National Academy of Sciences 101: 17306-17311

Umezawa T, Sugiyama N, Mizoguchi M, Hayashi S, Myouga F, Yamaguchi-Shinozaki K, Shinozaki K (2009) Type $2 \mathrm{C}$ protein phosphatases directly regulate abscisic acid-activated protein kinases in Arabidopsis. Proceedings of the National Academy of Sciences 106: 17588-17593

Walther E, Kerstin B, Hubert K (1999) Regulation of inducible nitric oxide synthase expression in b cells by environmental factors: heavy metals. Biochem J 338:695-700

Wang J, Lu K, Nie H, Zeng Q, Wu B, Qian J, Fang Z (2018) Rice nitrate transporter OsNPF7.2 positively regulates tiller number and grain yield. Rice 11(1). doi: 10.1186/s12284-018-0205-6

Xia X, Fan X, Wei J, Feng H, Qu H, Xie D, Miller AJ, Xu G (2015) Rice nitrate transporter OsNPF2.4 functions in low-affinity acquisition and long distance transport. Journal of Experimental Botany 66: 317-331 
Xing Y, Zhang Q (2010) Genetic and molecular bases of rice yield. Annu Annual Review of Plant Biology 61(1):421-442

Xu G, Fan X, Miller AJ (2012) Plant nitrogen assimilation and use efficiency. Annual Review of Plant Biology 63(1): 153-182

Yamaguchi-Shinozaki K, Shinozaki K (2006) Transcriptional regulatory networks in cellular responses and tolerance to dehydration and cold stresses. Annual Review of Plant Biology 57:781-803

Yamaya T, Kusano M (2014) Evidence supporting distinct functions of three cytosolic glutamine synthetases and two NADH-glutamate synthases in rice. Journal of Experimental Botany 65:5519-5525

Yang Z, Zhang Z, Zhang T, Fahad S, Cui K, Nie L, Peng S, Huang J (2017) The effect of season-long temperature increases on rice cultivars grown in the central and southern regions of China. Frontiers in Plant Science 8:1908 doi:10.3389/fpls.2017.01908

Zong W, Tang N, Yang J, Peng L, Ma S, Xu Y, Xiong L (2016) Feedback regulation of ABA signaling and biosynthesis by a bZIP transcription factor targets drought resistance related genes. Plant Physiology 171(4): pp.00469

\section{Supplemental Materials}

\section{Additional file 1}

Table S1. Primers used in this study.

SFig. 1 The comparison of nutrient content including nitrate among WT, sapk2 mutant lines and OE lines in rice seeds under reproductive-stage drought.

SFig. 2 SAPK2 affects seedling growth and root development under K deprivation.

SFig. 3 SAPK2 affects seedling growth and root development under P deprivation.

SFig. 1 The comparison of nutrient content including nitrate among WT, sapk2 mutant lines and $O E$ lines in rice seeds under reproductive-stage drought.

SFig. 2 SAPK2 affects seedling growth and root development under $\mathrm{K}$ deprivation. a-e Statistical analysis of shoot length (a),root length (b), dry weight of shoot (c), dry weight of root (d) and root number (e) among WT, sapk2 mutant lines and OE lines under K-deprived conditions. $\mathbf{f}$ Phenotypic analysis of seedlings at 31 DAG among WT, sapk2 mutant lines and OE lines under K-deprived conditions. Data in a-e are shown as means \pm SD $(n=20)$ from three replicates. A student's $t$-test was used to generate $P$ values;

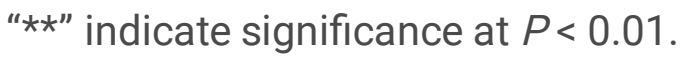


SFig. 3 SAPK2 affects seedling growth and root development under $\mathrm{P}$ deprivation. a-e Statistical analysis of shoot length (a),root length (b), dry weight of shoot (c), dry weight of root (d) and root number (e) among WT, sapk2 mutant lines and OE lines under P-deprived conditions. f Phenotypic analysis of seedlings at 31 DAG among WT, sapk2 mutant lines and $O E$ lines under P-deprived conditions. Data in a-e are shown as means $\pm \mathrm{SD}(n=20)$ from three replicates. A student's $t$-test was used to generate $P$ values;

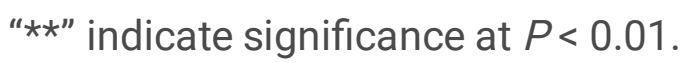

\section{Figures}

a

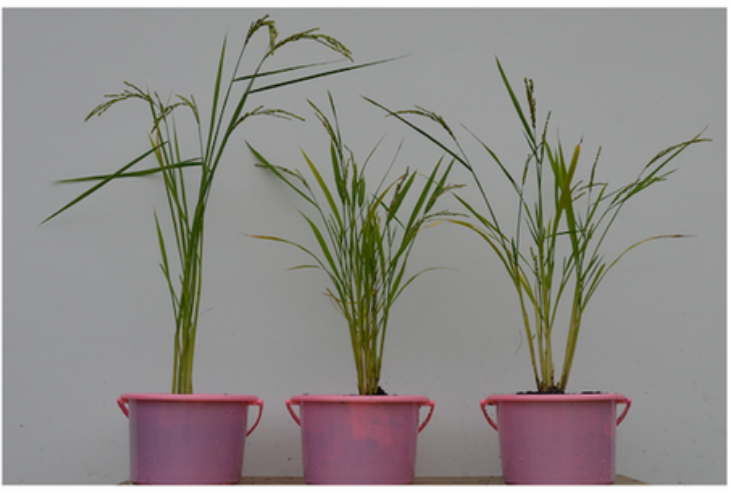

C

WT

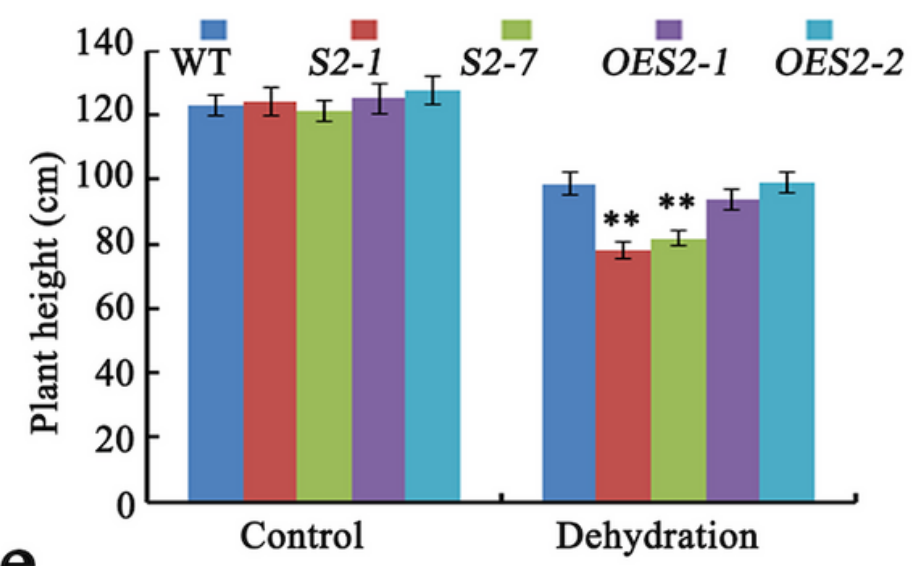

e

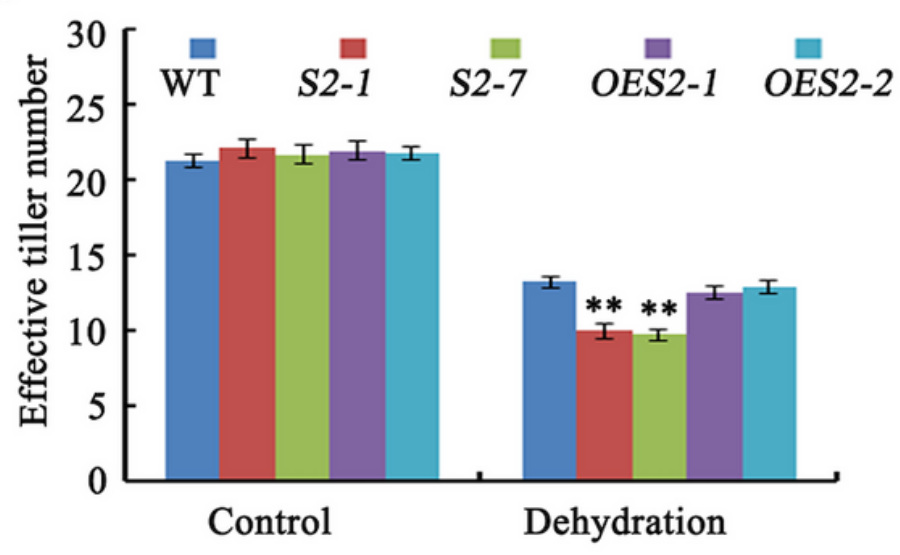

b

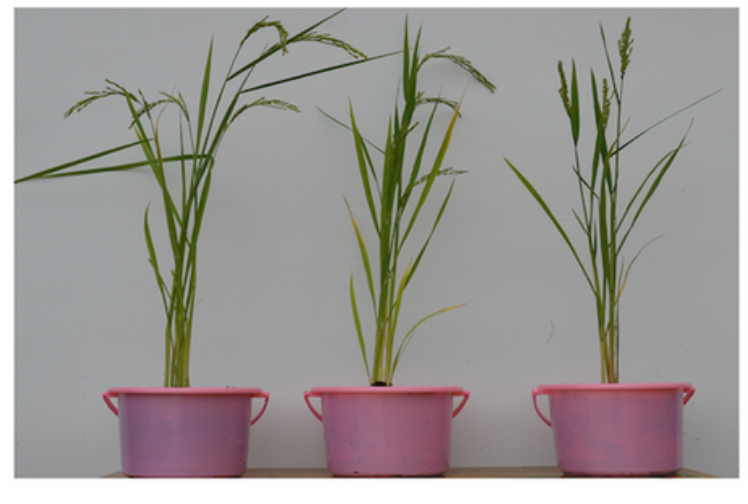

d

WT

OES2-1

OES2-2
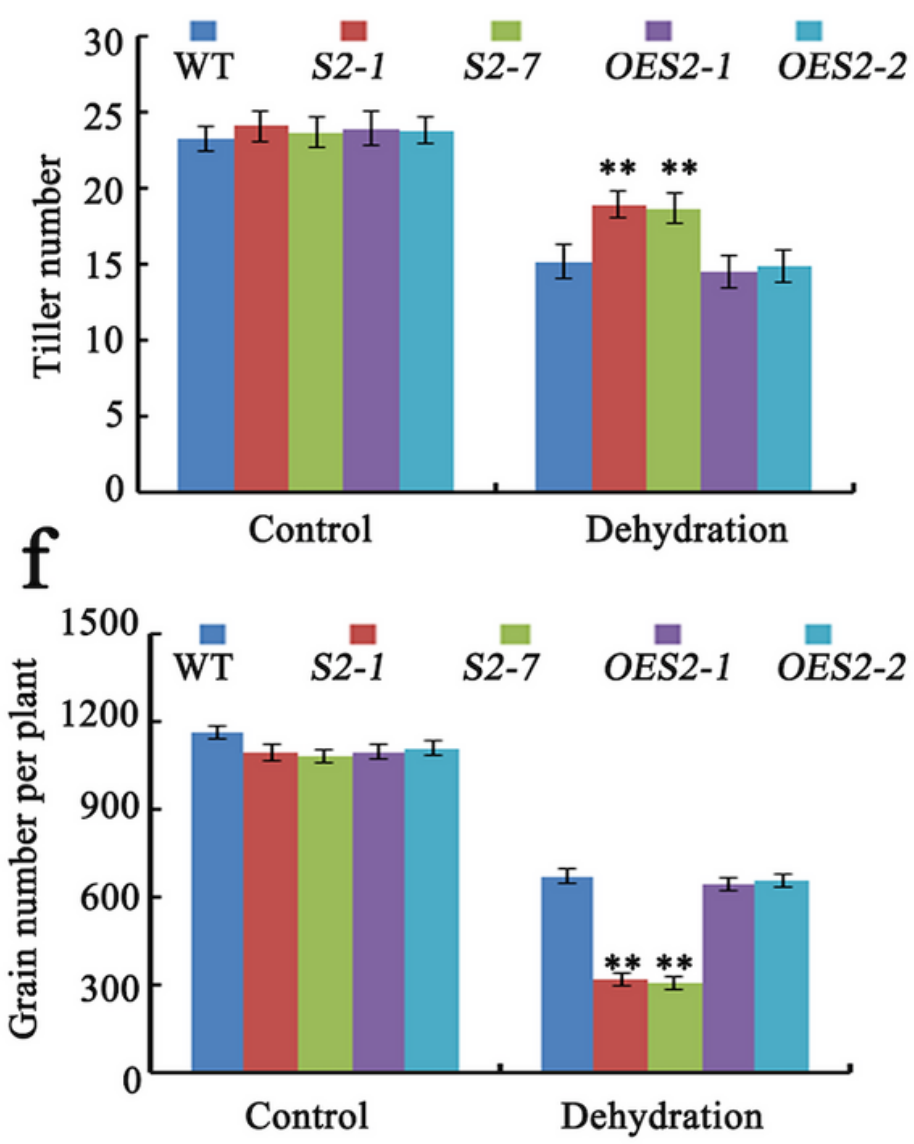
Figure 1

SAPK2 has large effects on plant height and grain number. a Phenotypes analysis of WT and sapk2 mutant lines at mature stage. b Phenotypes analysis of WT and OE lines at mature stage. c-f Comparison of agronomic traits including plant height (c), tiller number(d), effective tiller number(e), grain number per plant (f) among WT, sapk2 mutant lines and OE lines. Data in c-f are shown as means $\pm S D(n=20)$ from three replicates. A student's t-test was used to generate $P$ values; “**” indicate significance at $P<0.01$.

a
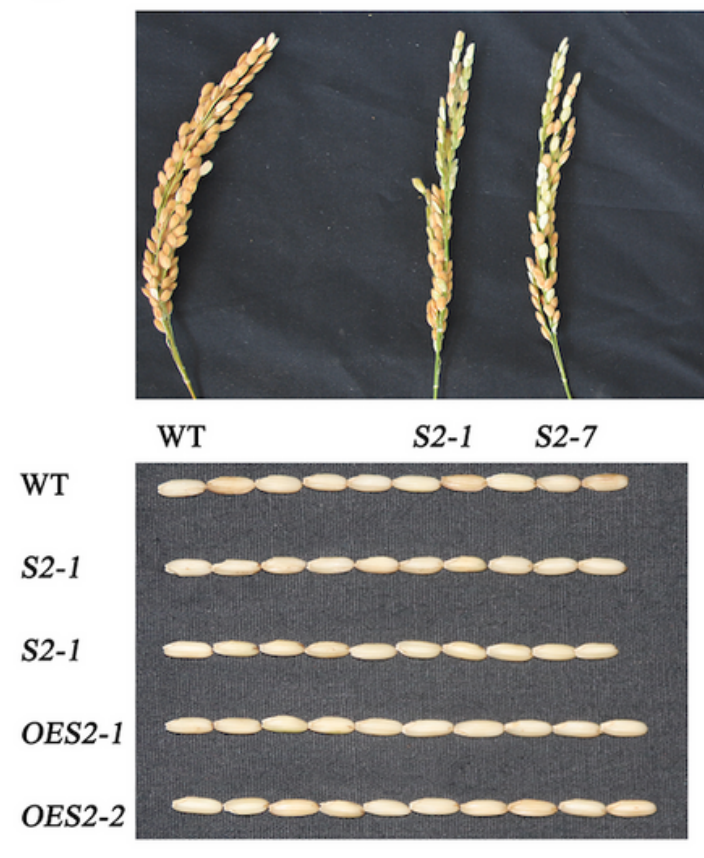

d
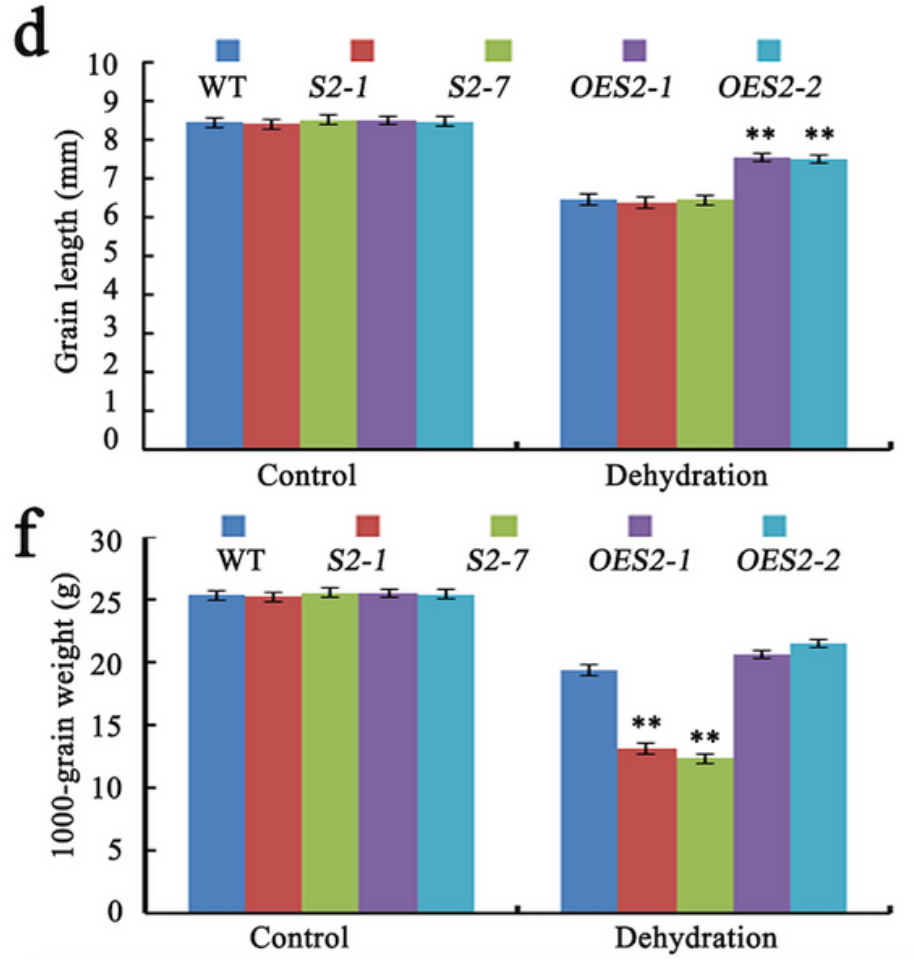

b
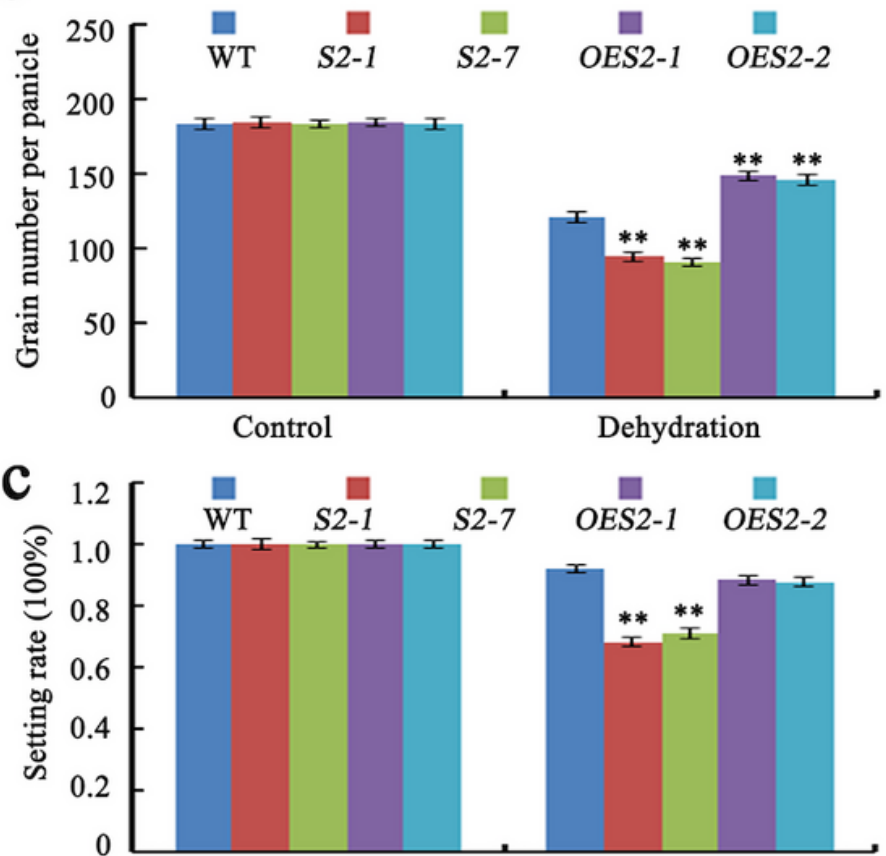

Control

Dehydration
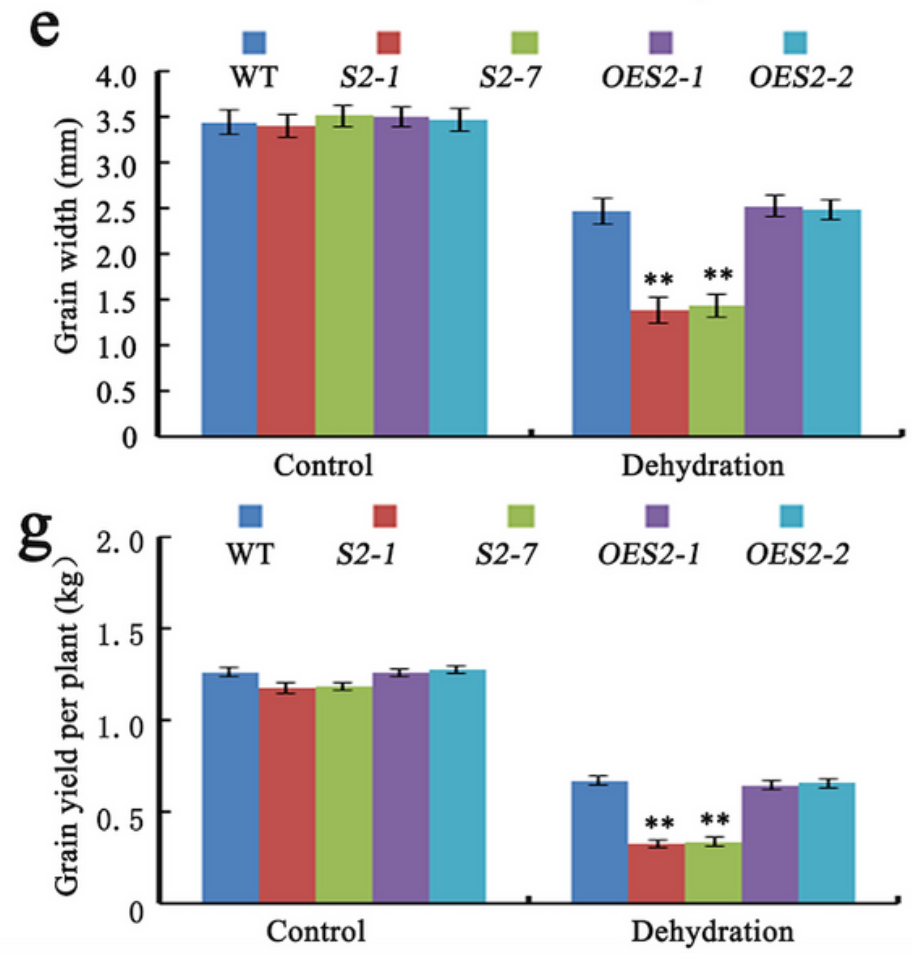

Figure 2 
SAPK2 has large effects on grain size and weight. a Panicle and grain phenotypes of WT, sapk2 mutant lines and $\mathrm{OE}$ lines. $b$-f Comparison of agronomic traits including grain number per panicle(b), setting rate (c), grain length (d), grain width (e), 1000-grain weight (f), grain yield per plant (g)among WT, sapk2 mutant lines and OE lines. Data in $b-f$ are shown as means $\pm S D(n=20)$ from three replicates. A student's t-test was used to generate $\mathrm{P}$ values; “**” indicate significance at $\mathrm{P}<0.01$.
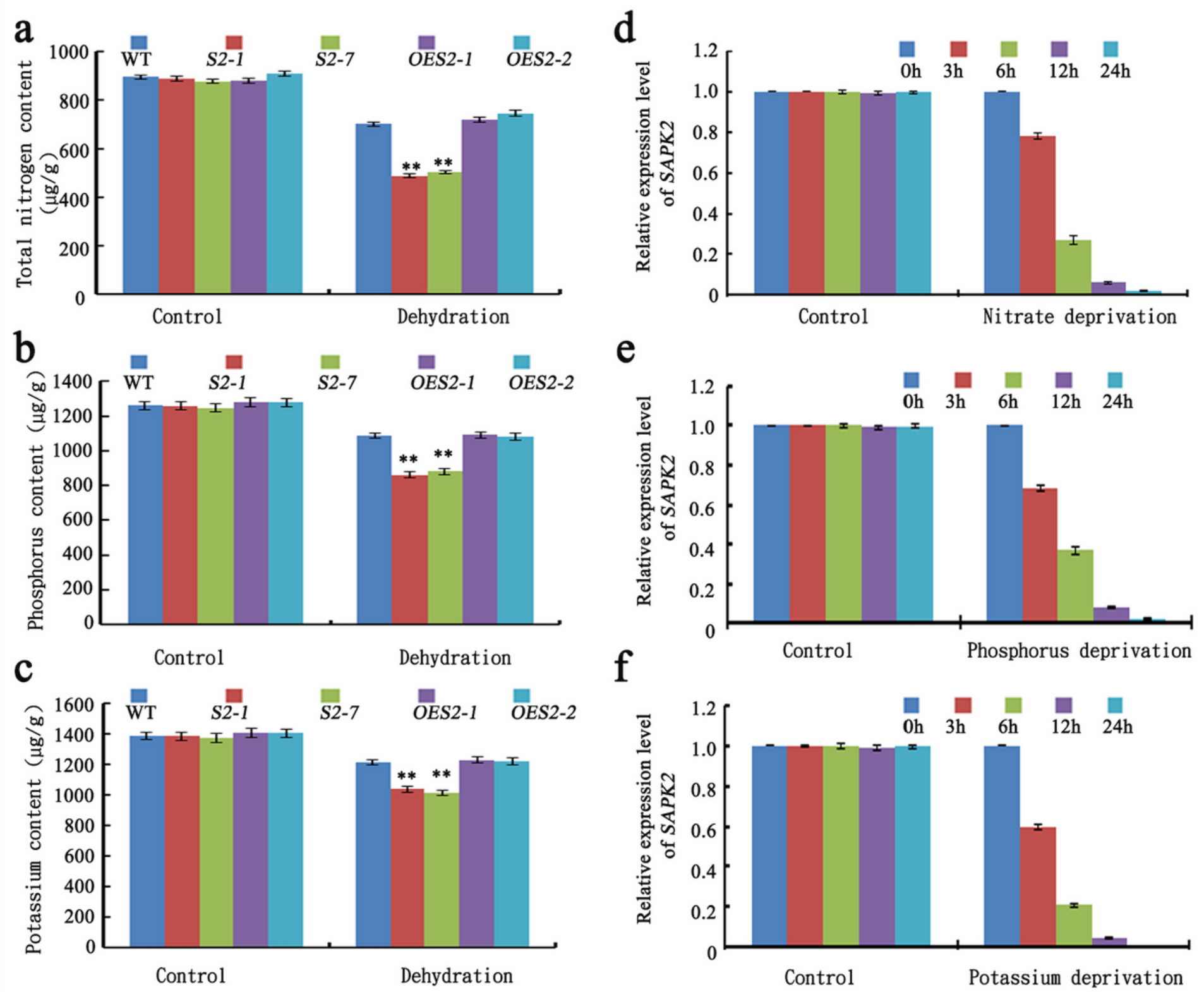

f

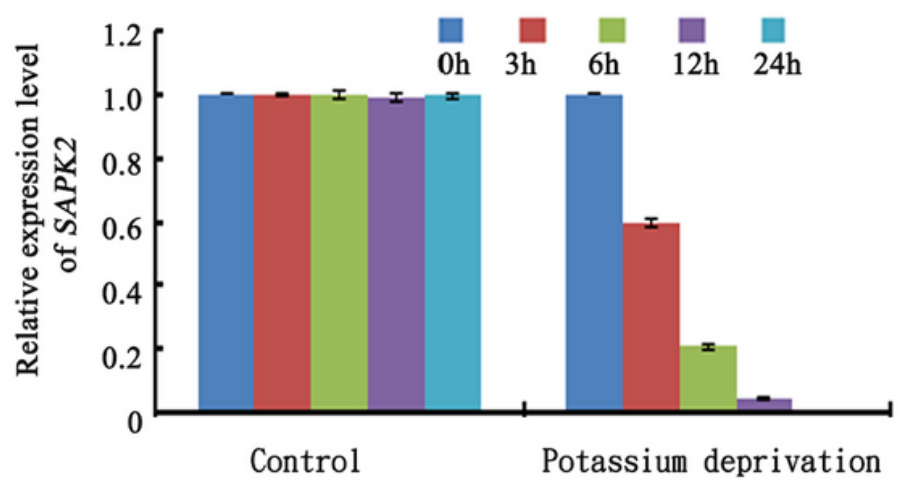

\section{Figure 3}

SAPK2 influenced Nitrate, phosphorus, potassium content in rice grains under reproductive-stage drought. a-c Comparison of nutrient content including total nitrogen (a), phosphorus (b), potassium (c) among WT, sapk2 mutant lines and OE lines in rice seeds under reproductive-stage drought. $d$-f The expression analysis of SAPK2 under $\mathrm{N}(\mathrm{d}), \mathrm{P}(\mathrm{e})$, and $\mathrm{K}(\mathrm{f})$ deprivation. Transcript accumulation was assessed by qRTPCR. Data are shown as means \pm SD $(n=20)$ from three replicates. A student's t-test was used to generate $P$ values; “**” indicate significance at $P<0.01$. 

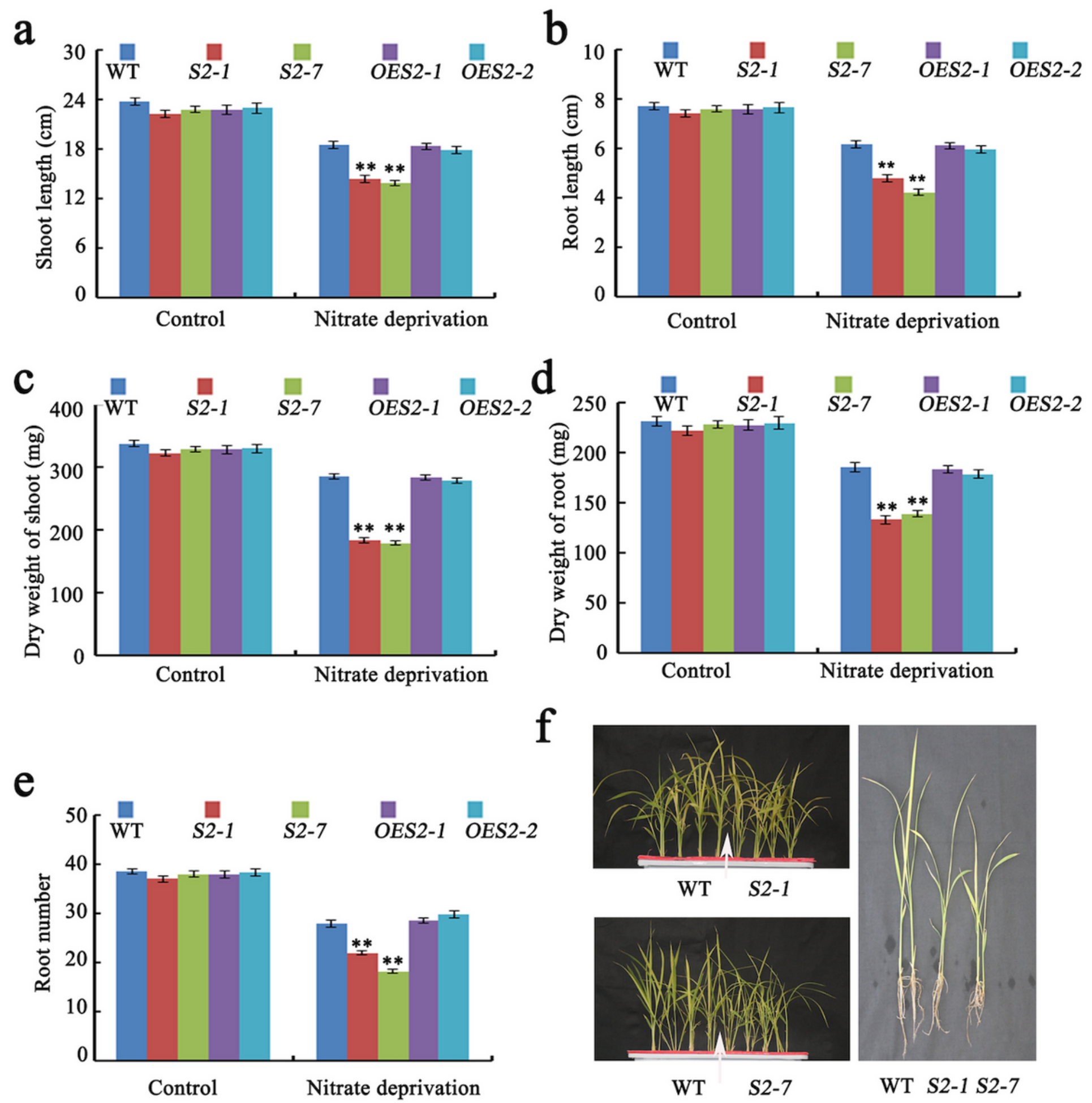

f

\section{Figure 4}

SAPK2 affects seedling growth and root development under $\mathrm{N}$ deprivation. a-e Statistical analysis of shoot length (a),root length (b), dry weight of shoot (c), dry weight of root (d) and root number (e) among WT, sapk2 mutant lines and OE lines under N-deprived conditions. $f$ Phenotypic analysis of seedlings at 31 DAG among WT, sapk2 mutant lines and OE lines under N-deprived conditions. Data in a-e are shown as means $\pm S D(n=20)$ from three replicates. A student's t-test was used to generate $P$ values; "**” indicate significance at $\mathrm{P}<0.01$. 
a

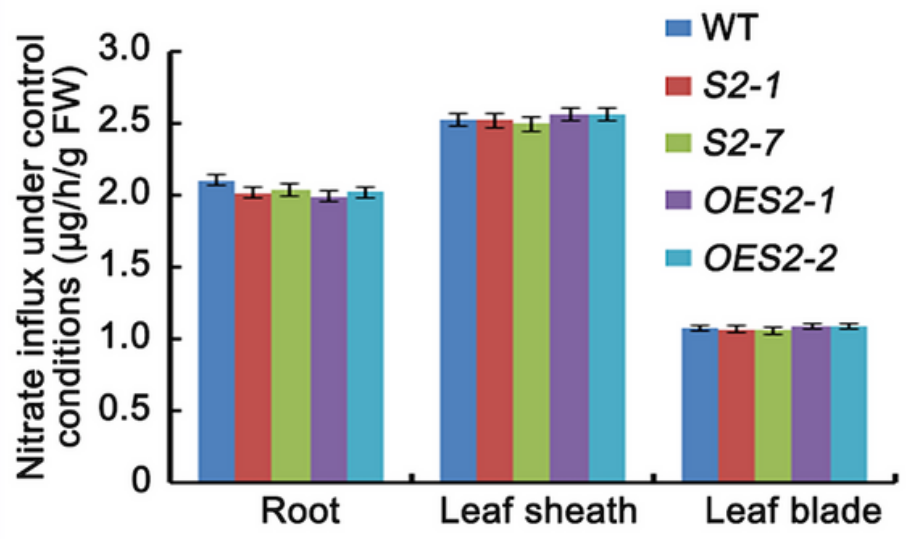

C

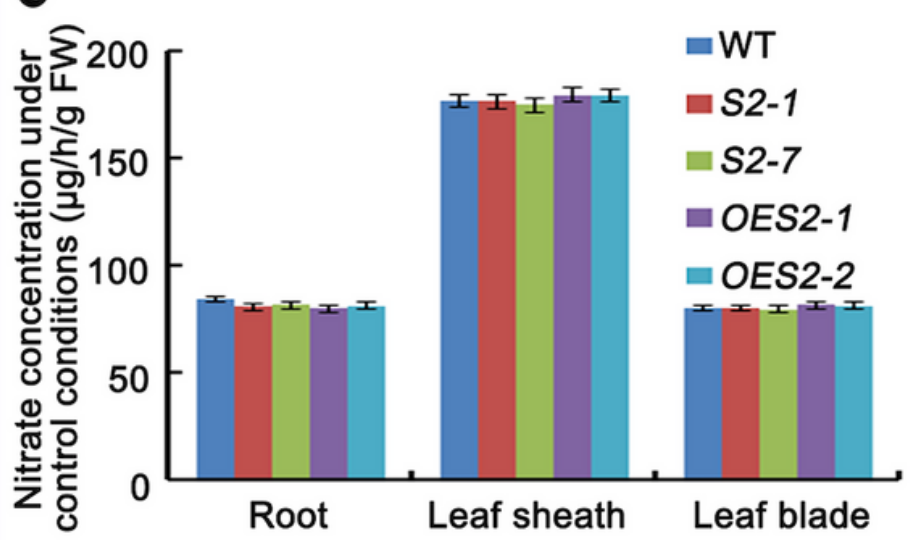

b

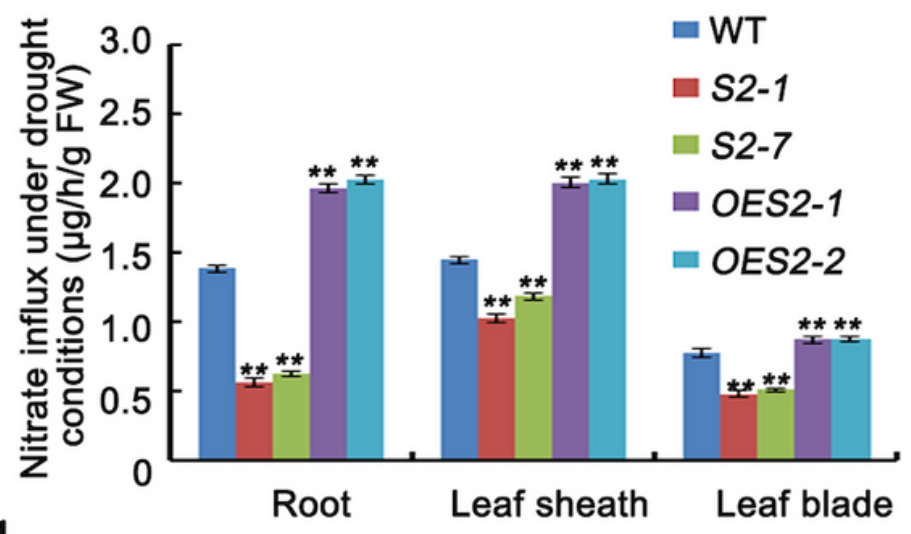

d

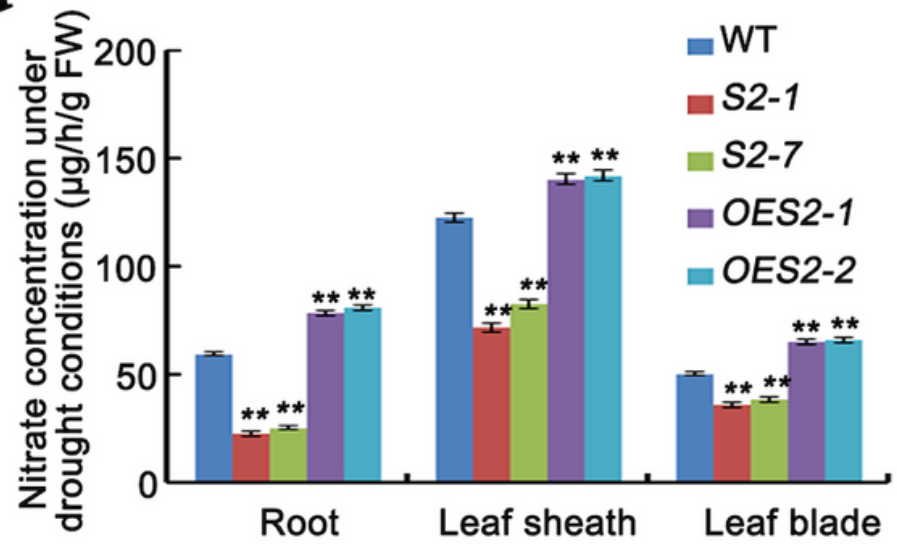

Figure 5

SAPK2 influenced the rate of NO3- influx rate and nitrate concentration. a-b Analysis of NO3- influx rate among WT, sapk2 mutant lines and OE lines under control conditions (a) and drought stress conditions (b). c-d Analysis of nitrate concentration among WT, sapk2 mutant lines and OE lines under control conditions (c) and drought stress conditions (d). Data are shown as means $\pm S D(n=20)$ from three

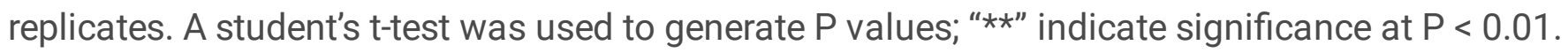


a

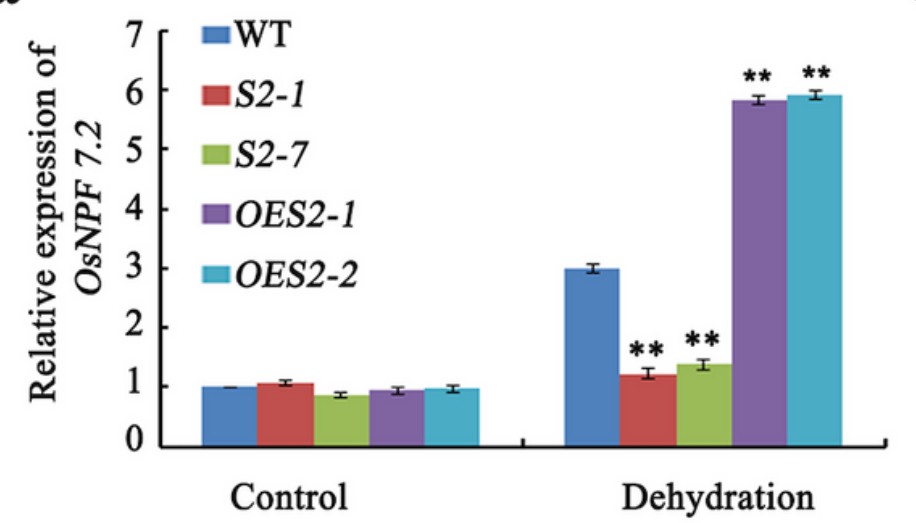

C

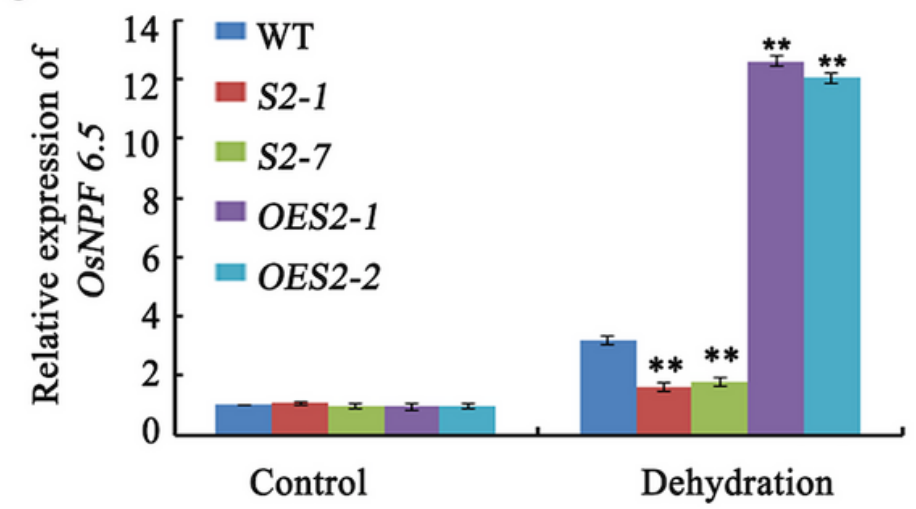

e

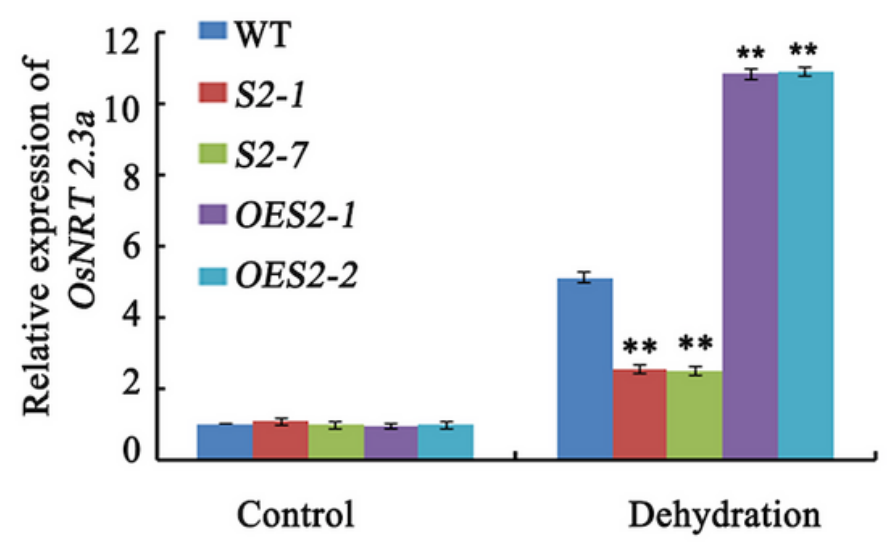

b

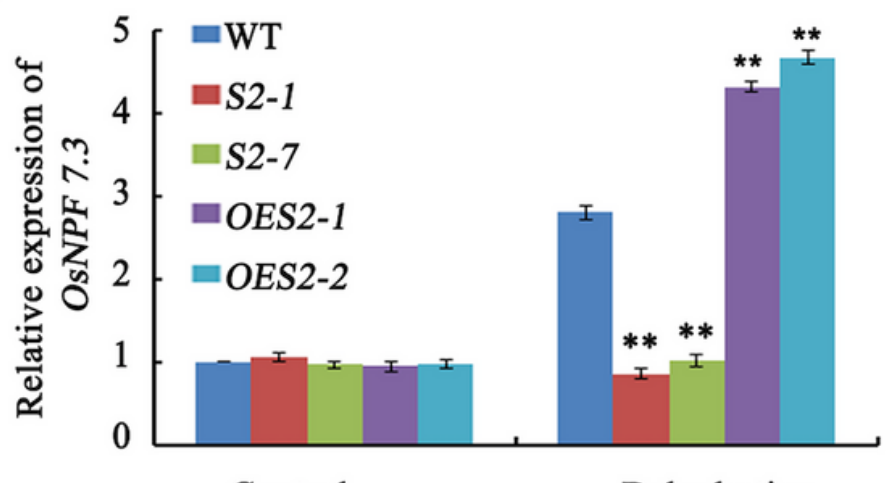

Control

Dehydration

d

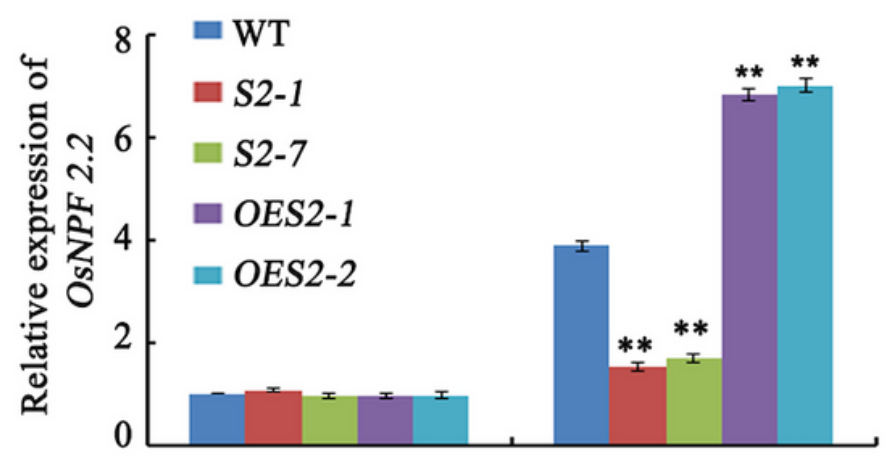

Control

Dehydration

f

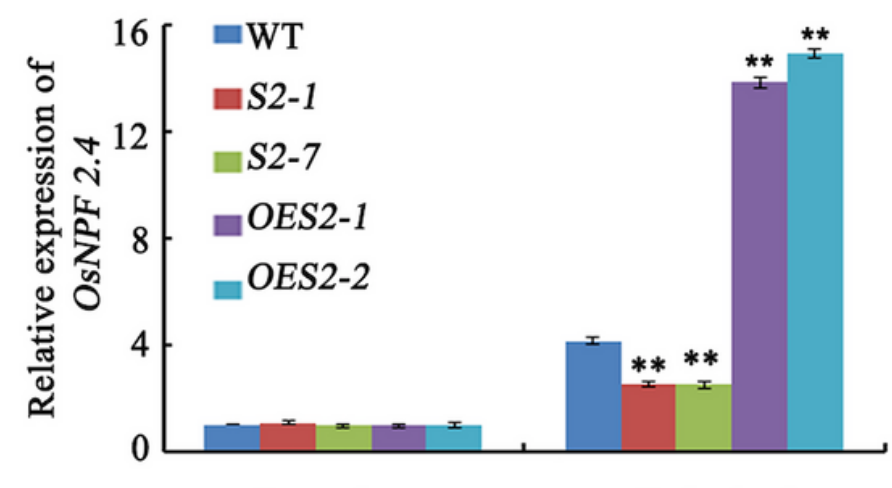

Control

Dehydration

Figure 6

Expression of genes involved in absorbing, transporting and assimilation of nitrate among WT, OE lines and sapk2 mutant lines. a-f Relative expression analysis of OsNPF7.2 (a), OsNPF7.3 (b), OsNPF5.6 (c), OsNPF2.2 (d), OsNRT2.3a (e) and OsNPF2.4 (f) among WT, sapk2 mutant lines and OE lines control conditions and drought stress conditions. Data are shown as means $\pm \operatorname{SD}(n=20)$ from three replicates. A student's t-test was used to generate $P$ values; “*ᄎ" indicate significance at $P<0.01$.

\section{Supplementary Files}


This is a list of supplementary files associated with this preprint. Click to download.

- Additionalfile1tables1.docx

- SFig2.tif

- SFig3.tif

- SFig1.tif 